INPLASY

PROTOCOL

To cite: Yu et al. Racial differences in the safety and efficacy of PCSK9 inhibitors in the treatment of

hyperlipidemia: A Systematic review and meta-analysis. Inplasy protocol 2021110047. doi:

10.37766/inplasy2021.11.0047

Received: 15 November 2021

Published: 15 November 2021

Corresponding author:

Yuqi Liu

ametuofo980869@163.com

Author Affiliation:

PLA General Hospital

Support: No.

Review Stage at time of this submission: Preliminary

searches.

Conflicts of interest:

None declared.

\section{Racial differences in the safety and efficacy of PCSK9 inhibitors in the treatment of hyperlipidemia: A Systematic review and meta-analysis}

Yu, Y1; Chen, L2; Zhang, H3; Fu, Z4; Liu, Q5; Zhao, H6; Liu, Y7; Chen, $Y^{8}$.

Review question / Objective: The purpose of this randomized controlled trial meta-analysis was to evaluate the efficacy and safety of PCSK9 inhibitors in the treatment of hyperlipidemia, especially the differences between different races.

Condition being studied: Statins are recommended as the first-line treatment of cardiovascular disease, which can significantly reduce the risk of cardiovascular disease. However, there are still a large number of patients who do not reach the optimal level of LDL-C, have the risk of residual CVD, or cannot tolerate statins, resulting in adverse reactions such as liver injury and myolysis. In recent years, PCSK9 has attracted much attention as a new target for the treatment of hypercholesterolemia. PCSK9 inhibitors have been shown to significantly reduce LDL-C levels in patients who do not meet statin treatment standards, reduce the occurrence of non fatal major adverse cardiac events (MACE), and are well tolerated. Blood lipid levels and atherosclerosis risk vary greatly among ethnic and geographical backgrounds. The genetic characteristics of Asian populations may lead to a slowdown in drug metabolism, including lipid-lowering drugs. Therefore, this study used meta-analysis to explore whether there were differences in the safety and efficacy of PCSK9 inhibitors among different races, especially Asian populations.

INPLASY registration number: This protocol was registered with the International Platform of Registered Systematic Review and Meta-Analysis Protocols (INPLASY) on 15 November 2021 and was last updated on 15 November 2021 (registration number INPLASY2021110047).

\section{INTRODUCTION}

Review question / Objective: The purpose of this randomized controlled trial metaanalysis was to evaluate the efficacy and safety of PCSK9 inhibitors in the treatment of hyperlipidemia, especially the differences between different races. 
Condition being studied: Statins are recommended as the first-line treatment of cardiovascular disease, which can significantly reduce the risk of cardiovascular disease. However, there are still a large number of patients who do not reach the optimal level of LDL-C, have the risk of residual CVD, or cannot tolerate statins, resulting in adverse reactions such as liver injury and myolysis. In recent years, PCSK9 has attracted much attention as a new target for the treatment of hypercholesterolemia. PCSK9 inhibitors have been shown to significantly reduce LDL-C levels in patients who do not meet statin treatment standards, reduce the occurrence of non fatal major adverse cardiac events (MACE), and are well tolerated. Blood lipid levels and atherosclerosis risk vary greatly among ethnic and geographical backgrounds. The genetic characteristics of Asian populations may lead to a slowdown in drug metabolism, including lipid-lowering drugs. Therefore, this study used metaanalysis to explore whether there were differences in the safety and efficacy of PCSK9 inhibitors among different races, especially Asian populations.

\section{METHODS}

Search strategy: The computer searched English databases such as PubMed, MEDLINE, Web of Science and BIOSIS, as well as Chinese databases such as CNKI, VIP and Wanfang, and collected randomized controlled studies on the efficacy and safety of PCSK9 inhibitors in the treatment of patients with hyperlipidemia or ASCVD or statin intolerance. The time limit was from the establishment of the database to November 2021, and the references of relevant literature were consulted. Search terms include: PCSK9, alirocumab, evolocumab, bococizumab, amg145, alnpcs02, bms-962476, ly3015014, rg7652, 1d05 lgG2, rn316, pf04950615, pf-05335810, regn727, sar236553, etc.

Participant or population: People with hyperlipidemia include ASCVD or statins intolerance or diabetes.
Intervention: PCSK9 inhibitors are used with or without statins as basic treatment

Comparator: The control group used statins, which may be the normal dose or maximum dose, and the LDLC still failed tomeet the standard: or used ezetimib, such as statin intolerant patients: or placebo.

Study designs to be included: Randomized controlled trials (RCTs) will be included.

Eligibility criteria: Inclusion criteria: 1) The study type was randomized controlled study; 2) The experimental group used PCSK9 inhibitors (with or without statins as basic treatment); The control group received statins, placebo or ezetimibe; 3 ) Outcome measures: the effectiveness index included the absolute value of the reduction of LDLC and / or the incidence of cardiovascular adverse events, and the safety index was the incidence of adverse events; 4) Double blind; 5) If the research results of different periods are published in the same research, the latest research data shall be included; 6) Including short-term research. Exclusion criteria: 1) duplicate or missing information, unable to extract key data information; 2) The subjects were children; 3) Meeting summaries, guidelines, case reports, letters, etc; 4) Animal tests; 5) Non Chinese and English literature.

Information sources: The computer searched English databases such as PubMed, MEDLINE, Web of Science and BIOSIS, as well as Chinese databases such as CNKI, VIP and Wanfang.

Main outcome(s): The effectiveness index includes the absolute value of LDLC reduction and / or the incidence of cardiovascular events, and the safety index is the incidence of adverse events.

Quality assessment / Risk of bias analysis: The two reviewers will independently assess the risk of deviation according to the following areas according to the recommendations in the Cochrane Manual: 1. Appropriate sequence generation; 2. 
Distribution concealment; 3. Blindness; 4. Incomplete result data and its processing method; 5. Report results selectively; 6 . Any other bias. The results of the bias assessment will show the low, high or unclear bias risk of each of the six items in each test in the form of figures and charts. Sensitivity analysis will be based on bias assessment to assess the robustness of the results.

Strategy of data synthesis: The relative risk (RR) is used as the effect index for the event incidence, and the mean difference (MD) is used as the effect index for the reduced value of LDLC. The point estimates and $95 \% \mathrm{Cl}$ of each effect quantity are given. We used Cochran's $Q$ test to assess differences between studies, and the $\mathbf{I}^{2}$ statistic was used to quantify the proportion of inconsistencies observed in the results. Values of $I^{2} \geq 50 \%$ and $P \leq 0.10$ indicated no heterogeneity among studies and a fixed-effect model was used for analysis. We also used Cochran's $Q$ test to calculate the heterogeneity between subgroups.

Subgroup analysis: Three subgroups will be analyzed: first, what is the difference between the safety and efficacy of PCSK9 inhibitors in the treatment of hyperlipidemia in different races, especially Asian and non Asians? Second, the difference in safety and efficacy between PCSK9 inhibitors in different research groups (such as familial hypercholesterolemia, high risk ASCVD or diabetes). Third, the net meta-analysis of different intervention measures, such as statins, PCSK9 inhibitors, statins and PCSK9 inhibitors, placebo, ezetimibe, etc.

Sensitivity analysis: Sensitivity analysis was performed for the results of the metaanalysis, and a funnel plot was drawn for publication bias analysis. If there was publication bias, we used the trim-and-fill method and Egger's test to verify whether publication bias affected the stability of the combined effect size.

Language: English and Chinese.
Keywords: PCSK9 inhibitor; Meta analysis; Randomized controlled trial; Hyperlipidemia; Racial differences.

Contributions of each author:

Author 1 - Yani Yu.

Email: 13752723933@163.com

Author 2 - Lei Chen.

Email: 317508951@qq.com

Author 3 - Honghong Zhang.

Email: diana_zhanghh@163.com

Author 4 - Zihao Fu.

Email: zihaofucaidiology@163.com

Author 5 - Qi Liu.

Email: Iqfern@163.com

Author 6 - Haijing Zhao.

Email: zhaohj0207@163.com

Author 7 - Yuqi Liu.

Email: ametuofo980869@163.com

Author 8 - Yundai Chen.

Email: cyundai@vip.163.com

Country(ies) involved: China. 